\title{
GLOBAL EXISTENCE AND BLOW-UP FOR A NON-NEWTON POLYTROPIC FILTRATION SYSTEM WITH NONLOCAL SOURCE
}

\author{
JUN ZHOU ${ }^{\otimes 1}$ and CHUNLAI MU ${ }^{1}$
}

(Received 17 April, 2007; revised 19 September, 2008)

\begin{abstract}
This paper deals the global existence and blow-up properties of the following nonNewton polytropic filtration system with nonlocal source,

$$
u_{t}-\Delta_{m, p} u=a \int_{\Omega} v^{\alpha}(x, t) d x, \quad v_{t}-\Delta_{n, q} v=b \int_{\Omega} u^{\beta}(x, t) d x .
$$

Under appropriate hypotheses, we prove that the solution either exists globally or blows up in finite time depending on the initial data and the relations between $\alpha \beta$ and $m n(p-1)(q-1)$. In the special case, $\alpha=n(q-1), \beta=m(p-1)$, we also give a criteria for the solution to exist globally or blow up in finite time, which depends on $a, b$ and $\zeta(x), \vartheta(x)$ as defined in our main results.
\end{abstract}

2000 Mathematics subject classification: primary 35K50; secondary 35K55, 35K65, 35B33.

Keywords and phrases: non-Newtonian polytropic system, nonlocal source, global existence, blow-up.

\section{Introduction}

In this paper, we consider the following doubly degenerate parabolic system with nonlocal source,

$$
\begin{aligned}
& u_{t}-\Delta_{m, p} u=a \int_{\Omega} v^{\alpha}(x, t) d x, \quad v_{t}-\Delta_{n, q} v=b \int_{\Omega} u^{\beta}(x, t) d x, \\
& (x, t) \in \Omega \times(0, T], \\
& u(x, t)=0, \quad v(x, t)=0, \quad(x, t) \in \partial \Omega \times(0, T], \\
& u(x, 0)=u_{0}(x), \quad v(x, 0)=v_{0}(x), \quad x \in \Omega \text {, }
\end{aligned}
$$

\footnotetext{
${ }^{1}$ School of Mathematics and Physics, Chongqing University, Chongqing, 400044, People's Republic of China; e-mail: zhoujun_math@hotmail.com.

(C) Australian Mathematical Society 2009, Serial-fee code 1446-1811/09 \$16.00
} 
where for $k>0, \gamma>2$ and $N \geq 1$,

$$
\Delta_{k, \gamma} \Theta=\nabla \cdot\left(\left|\nabla \Theta^{k}\right|^{\gamma-2} \cdot \nabla \Theta^{k}\right), \quad \nabla \Theta^{k}=k \Theta^{k-1}\left(\Theta_{x_{1}}, \ldots, \Theta_{x_{N}}\right),
$$

$\Omega \subset \mathbb{R}^{N}(N \geq 1)$ is a bounded domain with smooth boundary $\partial \Omega ; m, n \geq 1, p, q>2$, $\alpha, \beta>0$ are parameters; and $a$ and $b$ are positive constants.

Throughout this paper, we use the notation $Q_{T}=\Omega \times(0, T], S_{T}=\partial \Omega \times[0, T]$, $T>0$, and make the following assumption on initial data.

ASSUMPTION 1.1. The nonnegative initial data satisfies compatibility conditions and $u_{0}^{m}(x) \in C(\bar{\Omega}) \cap W_{0}^{1, p}(\Omega), v_{0}^{n}(x) \in C(\bar{\Omega}) \cap W_{0}^{1, q}(\Omega)$, and $\nabla u_{0}^{m} \cdot v<0, \nabla v_{0}^{n} \cdot v<0$ on the boundary $\partial \Omega$, where $v$ is unit outer normal vector on $\partial \Omega$.

Parabolic systems such as (1.1) appear in population dynamics, chemical reactions, heat transfer equations and so on. In particular, Equations (1.1) may be used to describe the nonstationary flows in a porous medium of fluids with a power dependence of the tangential stress on the velocity of displacement under polytropic conditions. In this case, Equations (1.1) are called the non-Newtonian polytropic filtration equations (see [16, 25, 27] and references therein). The problems with the nonlinear reaction term and nonlinear diffusion include blow-up and global existence conditions of solutions, blow-up rates and blow-up sets, etc. (see the surveys $[4,14,17,22])$. Here, we say that a solution blows up in finite time if the solution becomes unbounded (in the sense of maximum norm) at that time.

System (1.1) has been studied by many authors. For $p=q=2$, this system is known as the porous medium equations (see [6, 21, 29] for nonlinear boundary conditions, see $[3,11,12]$ for local nonlinear reaction terms and see $[1,5,9,10,20]$ for nonlocal nonlinear reaction terms).

In [20], Li and Xie considered the following problem,

$$
\begin{aligned}
u_{t}-\Delta u^{m} & =a \int_{\Omega} u^{p} d x, & & (x, t) \in \Omega \times(0, T], \\
u(x, t) & =0, & & x \in \partial \Omega \times(0, T], \\
u(x, 0) & =u_{0}(x), & & x \in \Omega,
\end{aligned}
$$

and obtained that the solution either exists globally or blows up in finite time under appropriate assumptions. Furthermore, if $p>m$, they also obtained the blow-up rate.

Recently, in [9], Du generalized (1.2) to a system, and studied the following problem,

$$
\begin{aligned}
u_{t}-\Delta u^{m} & =\int_{\Omega} v^{p} d x, & v_{t}-\Delta v^{n} & =\int_{\Omega} u^{q} d x, & & (x, t) \in \Omega \times(0, T], \\
u(x, t) & =0, & v(x, t) & =0, & & (x, t) \in \partial \Omega \times(0, T], \\
u(x, 0) & =u_{0}(x), & v(x, 0) & =v_{0}(x), & & x \in \Omega .
\end{aligned}
$$


Similar to [20], he also obtained the criteria for a solution to exist globally or blow up in finite time. Moreover, if $p>n, q>m$, he also obtained the blow-up rates under the monotone assumption for initial data.

When $m=n=1$, problem (1.1) is called the Laplace equations (see [13, 30] for nonlinear boundary conditions, see [15, 18, 24, 28] for local nonlinear reaction terms and see [19] for nonlocal nonlinear reaction terms).

In [19], Li and Xie considered the following problem,

$$
\begin{aligned}
u_{t}-\nabla \cdot\left(|\nabla u|^{p-2}\right) & =\int_{\Omega} u^{q} d x, & & (x, t) \in \Omega \times(0, T], \\
u(x, t) & =0, & & x \in \partial \Omega \times(0, T], \\
u(x, 0) & =u_{0}(x), & & x \in \Omega,
\end{aligned}
$$

and they obtained that the solution $u$ exists globally if $q<p-1 ; u$ blows up in finite time if $q>p-1$ and $u_{0}(x)$ is large enough.

For general $m, n \geq 1, p, q>2, \alpha, \beta>0$, problem (1.1) is called non-Newton polytropic filtration system, and only the case of nonlinear boundary condition has been considered extensively (see [23, 26, 31]). However, it seems that the case of local (nonlocal) nonlinear reaction terms is less studied.

In [23], Sun and Wang considered the following doubly degenerate equation,

$$
\begin{aligned}
\left(u^{m}\right)_{t} & =\Delta_{1, p} u, & & (x, t) \in \Omega \times(0, T], \\
|\nabla u|^{p-2} \nabla u \cdot v & =u^{\alpha}, & & x \in \partial \Omega \times(0, T], \\
u(x, 0) & =u_{0}(x), & & x \in \Omega,
\end{aligned}
$$

by using upper and lower solution methods, they proved that all positive solutions of (1.5) exist globally if and only if $\alpha \leq m$ when $m \leq p-1$, or $\alpha \leq(p-1)(m+1) / p$ when $m>p-1$.

In [26], Wang considered the system of (1.5) in one dimension,

$$
\begin{aligned}
\left(u^{m}\right)_{t} & =\left(\left|u_{x}\right|^{p-2} u_{x}\right)_{x}, & \left(v^{n}\right)_{t} & =\left(\left|v_{x}\right|^{q-2} v_{x}\right)_{x}, & & (x, t) \in(0,1) \times(0, T], \\
u_{x}(0, t) & =0, & u_{x}(1, t) & =a u^{\alpha} v^{r}(1, t), & & t \in(0, T], \\
v_{x}(0, t) & =0, & v_{x}(1, t) & =b u^{\beta} v^{s}(1, t), & & t \in(0, T], \\
u(x, 0) & =u_{0}(x), & v(x, 0) & =v_{0}(x), & & x \in(0,1) .
\end{aligned}
$$

He obtained that all positive solutions of (1.6) exist globally if and only if

$$
\begin{gathered}
\alpha<\min \left\{\frac{m}{p-1}, \frac{m+1}{p}\right\}, \quad s<\min \left\{\frac{n}{q-1}, \frac{n+1}{q}\right\}, \\
\beta r \leq\left(\min \left\{\frac{m}{p-1}, \frac{m+1}{p}\right\}-\alpha\right)\left(\min \left\{\frac{n}{q-1}, \frac{n+1}{q}\right\}-s\right) .
\end{gathered}
$$

Motivated by the references cited above, in this paper we investigate the blow-up properties of solutions to the problem (1.1) and extend the results of $[9,19,20]$ to more generalized cases. Our main results are stated as follows. 
THEOREM 1.2. Suppose that the initial data $\left(u_{0}(x), v_{0}(x)\right)$ satisfies the Assumption 1.1, then the solution of Problem (1.1) exists globally if one of the following conditions holds:

(i) $\alpha \beta<m n(p-1)(q-1)$;

(ii) $\alpha \beta=m n(p-1)(q-1)$ and $\|\Omega\|$ is sufficiently small, where $\|\Omega\|$ is the measure of $\Omega$;

(iii) $\alpha \beta>m n(p-1)(q-1)$ and the initial value is sufficiently small.

TheORem 1.3. Suppose that the initial data $\left(u_{0}(x), v_{0}(x)\right)$ satisfies the Assumption 1.1, then the solution of Problem (1.1) blows up in finite time if one of the following conditions holds:

(i) $\alpha \beta>m n(p-1)(q-1)$ and the initial is sufficiently large;

(ii) $\alpha \beta=m n(p-1)(q-1)$ and $\Omega$ contains a sufficiently large ball.

Next, we consider a special case $\alpha=n(q-1), \beta=m(p-1)$.

THEOREM 1.4. Suppose that the initial data $\left(u_{0}(x), v_{0}(x)\right)$ satisfies the Assumption 1.1, and that $\alpha=n(q-1), \beta=m(p-1)$.

(i) If $\lambda \mu \leq(a b)^{-1}$, then the solution of Problem (1.1) exists globally.

(ii) If $\lambda \mu>(a b)^{-1}$, then the solution of Problem (1.1) blows up in finite time, where

$$
\lambda=\int_{\Omega} \zeta^{m(p-1)}(x) d x, \quad \mu=\int_{\Omega} \vartheta^{n(q-1)}(x) d x,
$$

and $\zeta(x), \vartheta(x)$ are the unique solution of the following elliptic equation (see $[2,7])$,

$$
\left\{\begin{array} { l l } 
{ - \Delta _ { m , p } \zeta = 1 , } & { x \in \Omega , } \\
{ \zeta = 0 , } & { x \in \partial \Omega , }
\end{array} \quad \left\{\begin{array}{ll}
-\Delta_{n, q} \vartheta=1, & x \in \Omega, \\
\vartheta=0, & x \in \partial \Omega .
\end{array}\right.\right.
$$

This paper is organized as follows. In Section 2, we give some preliminaries, which form the basis of the proof of our theorems. The proof of Theorem 1.2 is the subject of Section 3. In Section 4, we consider the blow-up properties of Problem (1.1) and give the proof of Theorem 1.3. Finally, in Section 5, we consider a special case of Problem (1.1) and give the proof of Theorem 1.4.

\section{Preliminaries}

As it is well known that degenerate equations need not have classical solutions, we give a precise definition of a weak solution for Problem (1.1). 
Definition 2.1. A pair of functions $(u(x, t), v(x, t))$ is called an upper (lower) solution of Problem (1.1) in $\bar{Q}_{T} \times \bar{Q}_{T}$ if and only if

$$
\begin{gathered}
u^{m}(x, t) \in C\left(0, T ; L^{\infty}(\Omega)\right) \cap L^{p}\left(0, T ; W_{0}^{1, p}(\Omega)\right), \\
v^{n}(x, t) \in C\left(0, T ; L^{\infty}(\Omega)\right) \cap L^{q}\left(0, T ; W_{0}^{1, q}(\Omega)\right), \\
u_{t} \in L^{2}\left(0, T ; L^{2}(\Omega)\right), \quad v_{t} \in L^{2}\left(0, T ; L^{2}(\Omega)\right), \\
u(x, 0)=u_{0}(x), \quad v(x, 0)=v_{0}(x),
\end{gathered}
$$

and the following inequalities

$$
\begin{aligned}
& \int_{\Omega} u\left(x, t_{2}\right) \psi\left(x, t_{2}\right) d x-\int_{\Omega} u\left(x, t_{1}\right) \psi\left(x, t_{1}\right) d x \\
& \geq(\leq) \int_{t_{1}}^{t_{2}} \int_{\Omega} u \psi_{t} d x d t-\int_{t_{1}}^{t_{2}} \int_{\Omega}\left|\nabla u^{m}\right|^{p-2} \nabla u^{m} \cdot \nabla \psi d x d t \\
& \quad+a \int_{t_{1}}^{t_{2}} \int_{\Omega} \psi(x, t)\left(\int_{\Omega} v^{\alpha}(x, t) d x\right) d x d t \\
& \int_{\Omega} v\left(x, t_{2}\right) \psi\left(x, t_{2}\right) d x-\int_{\Omega} v\left(x, t_{1}\right) \psi\left(x, t_{1}\right) d x \\
& \geq(\leq) \int_{t_{1}}^{t_{2}} \int_{\Omega} v \psi_{t} d x d t-\int_{t_{1}}^{t_{2}} \int_{\Omega}\left|\nabla v^{n}\right|^{q-2} \nabla v^{n} \cdot \nabla \psi d x d t \\
& \quad+b \int_{t_{1}}^{t_{2}} \int_{\Omega} \psi(x, t)\left(\int_{\Omega} u^{\beta}(x, t) d x\right) d x d t
\end{aligned}
$$

hold for all $0<t_{1}<t_{2}<T$, where $\psi(x, t) \geq 0 \in C^{1,1}\left(\bar{Q}_{T}\right)$ such that $\psi(x, T)=0$ and $\psi(x, t)=0$ on $S_{T}$. In particular, $(u(x, t), v(x, t))$ is called a weak solution of (1.1) if it is both a weak upper and a weak lower solution.

The local existence of weak solutions to Problem (1.1) under Assumption 1.1 and the following comparison principle is standard (see [8, 16, 25, 27]).

COMPARISOn PRInciple. Suppose that $(\underline{u}(x, t), \underline{v}(x, t))$ and $(\bar{u}(x, t), \bar{v}(x, t))$ are the lower and upper solution of Problem (1.1) on $\bar{Q}_{T} \times \bar{Q}_{T}$, respectively. Then $(\underline{u}(x, t), \underline{v}(x, t)) \leq(\bar{u}(x, t), \bar{v}(x, t))$ almost everywhere on $\bar{Q}_{T} \times \bar{Q}_{T}$.

In order to study the globally existing solutions to Problem (1.1), we need to study the following elliptic system

$$
\begin{array}{ll}
-\Delta_{k, \gamma} \Theta=1, & x \in \Omega, \\
\Theta=1, & x \in \partial \Omega,
\end{array}
$$

where $\Delta_{k, \gamma} \Theta$ is defined in (1.1), and we obtain the following lemma. 
LEMMA 2.2. Problem (2.1) has a unique solution $\Theta(x)$, and satisfies the following relations,

$$
\Theta(x)>1 \text { in } \Omega, \quad \nabla \Theta \cdot v<0 \quad \text { on } \partial \Omega, \quad \sup _{x \in \Omega} \Theta=M<+\infty,
$$

where $M$ is a positive constant.

Proof. Set $\Theta^{k}=\Phi$, then $\Phi$ satisfies the following equation

$$
\begin{array}{ll}
-\nabla \cdot\left(|\nabla \Phi|^{\gamma-2} \nabla \Phi\right)=1, & x \in \Omega, \\
\Phi=1, & x \in \partial \Omega .
\end{array}
$$

Next, let $\Psi=\Phi-1$, then the following equation reads

$$
\begin{array}{ll}
-\nabla \cdot\left(|\nabla \Psi|^{\gamma-2} \nabla \Psi\right)=1, & x \in \Omega, \\
\Psi=0, & x \in \partial \Omega .
\end{array}
$$

Then from [2, 7], we obtain that Problem (2.3) has a unique solution $\Psi(x)$, and satisfies the following relations,

$$
\Psi(x)>0 \text { in } \Omega, \quad \nabla \Psi \cdot v<0 \text { on } \partial \Omega, \quad \sup _{x \in \Omega} \Psi=M^{\prime}<+\infty,
$$

where $M^{\prime}$ is a positive constant. Since $\Theta=(\Psi+1)^{1 / k}$, the conclusion of Lemma 2.2 follows directly.

\section{Global existence of a solution}

In this section, we investigate the global existence property of the solutions to Problem (1.1) and prove Theorem 1.2. The main method is constructing a globally upper solution and using comparison principle to achieve our purpose.

PROOF OF THEOREM 1.2. Let $\varphi(x)$ and $\psi(x)$ be the unique solution of the following elliptic problem

$$
\left\{\begin{array} { l l } 
{ - \Delta _ { m , p } \varphi = 1 , } & { x \in \Omega , } \\
{ \varphi = 1 , } & { x \in \partial \Omega , }
\end{array} \quad \left\{\begin{array}{ll}
-\Delta_{n, q} \psi=1, & x \in \Omega \\
\psi=1, & x \in \partial \Omega .
\end{array}\right.\right.
$$

Then from Lemma 2.2, we obtain the following relations

$$
\begin{array}{ll}
\varphi(x), \psi(x)>1 \text { in } \Omega, & \nabla \varphi \cdot v, \nabla \psi \cdot v<0 \text { on } \partial \Omega, \\
\sup _{x \in \Omega} \varphi=M_{1}<+\infty, & \sup _{x \in \Omega} \psi=M_{2}<+\infty,
\end{array}
$$

where $M_{1}, M_{2}$ are positive constants. 
Let $\bar{u}(x, t)=\Lambda_{1} \varphi(x), \bar{v}(x, t)=\Lambda_{2} \psi(x)$, where $\Lambda_{1}, \Lambda_{2}>0$ will be determined later. Then with a direct computation we obtain

$$
\bar{u}_{t}-\Delta_{m, p} \bar{u}=\Lambda_{1}^{m(p-1)}, \quad \bar{v}_{t}-\Delta_{n, q} \bar{v}=\Lambda_{2}^{n(q-1)},
$$

and

$$
a \int_{\Omega} \bar{v}^{\alpha} d x \leq a\|\Omega\| M_{2}^{\alpha} \Lambda_{2}^{\alpha}, \quad b \int_{\Omega} \bar{u}^{\beta} d x \leq b\|\Omega\| M_{1}^{\beta} \Lambda_{1}^{\beta} .
$$

So, $(\bar{u}(x, t), \bar{v}(x, t))$ is an upper solution of Problem (1.1), if

$$
\begin{gathered}
\Lambda_{1}^{m(p-1)} \geq a\|\Omega\| M_{2}^{\alpha} \Lambda_{2}^{\alpha}, \quad \Lambda_{2}^{n(q-1)} \geq b\|\Omega\| M_{1}^{\beta} \Lambda_{1}^{\beta}, \\
\left.\bar{u}(x, t)\right|_{\partial \Omega} \geq 0,\left.\quad \bar{v}(x, t)\right|_{\partial \Omega} \geq 0, \quad \bar{u}(x, 0) \geq u_{0}(x), \quad \bar{v}(x, 0) \geq v_{0}(x) .
\end{gathered}
$$

Next we prove (3.1) in three cases.

(i) When $\alpha \beta<m n(p-1)(q-1)$, if we choose $\Lambda_{1}, \Lambda_{2}$ large enough such that

$$
\begin{aligned}
& \Lambda_{1}>\max \left\{\max _{x \in \bar{\Omega}} u_{0}(x),\left(a b^{\frac{\alpha}{n(q-1)}}\|\Omega\|^{\frac{n(q-1)+\alpha}{n(q-1)}} M_{1}^{\frac{\alpha \beta}{n(q-1)}} M_{2}^{\alpha}\right)^{\frac{n(q-1)}{m n(p-1)(q-1)-\alpha \beta}}\right\}, \\
& \Lambda_{2}>\max \left\{\max _{x \in \bar{\Omega}} v_{0}(x),\left(a^{\frac{\beta}{m(p-1)}} b\|\Omega\|^{\frac{m(p-1)+\beta}{m(p-1)}} M_{1}^{\beta} M_{2}^{\frac{\alpha \beta}{m(p-1)}}\right)^{\frac{m(p-1)}{m n(p-1)(q-1)-\alpha \beta}}\right\},
\end{aligned}
$$

then (3.1) holds.

(ii) When $\alpha \beta=m n(p-1)(q-1)$, we can choose $\Lambda_{1}, \Lambda_{2}$ large enough such that

$$
\Lambda_{1}>\max _{x \in \bar{\Omega}} u_{0}(x), \quad \Lambda_{2}>\max _{x \in \bar{\Omega}} v_{0}(x),
$$

and $\|\Omega\|$ small enough such that

$$
\|\Omega\| \leq \min \left\{\left(a b^{\frac{\alpha}{n(q-1)}} M_{1}^{\frac{\alpha \beta}{n(q-1)}} M_{2}^{\alpha}\right)^{\frac{n(1-q)}{n(q-1)+\alpha}},\left(a^{\frac{\beta}{m(p-1)}} b M_{1}^{\beta} M_{2}^{\frac{\alpha \beta}{m(p-1)}}\right)^{\frac{m(1-p)}{m(p-1)+\beta}}\right\},
$$

then (3.1) holds.

(iii) When $\alpha \beta>m n(p-1)(q-1)$, we can take $\Lambda_{1}, \Lambda_{2}$ small enough such that

$$
\begin{aligned}
& \Lambda_{1}<\left(a b^{\frac{\alpha}{n(q-1)}}\|\Omega\|^{\frac{n(q-1)+\alpha}{n(q-1)}} M_{1}^{\frac{\alpha \beta}{n(q-1)}} M_{2}^{\alpha}\right)^{\frac{n(1-q)}{\alpha \beta-m n(p-1)(q-1)}}, \\
& \Lambda_{2}<\left(a^{\frac{\beta}{m(p-1)}} b\|\Omega\|^{\frac{m(p-1)+\beta}{m(p-1)}} M_{1}^{\beta} M_{2}^{\frac{\alpha \beta}{m(p-1)}}\right)^{\frac{m(1-p)}{\alpha \beta-m n(p-1)(q-1)}} .
\end{aligned}
$$

Furthermore, if the initial data is sufficiently small such that $u_{0}(x) \leq \Lambda_{1}$ and $v_{0}(x) \leq \Lambda_{2}$, then (3.1) holds. The proof of Theorem 1.2 is complete. 


\section{Blow-up of a solution}

In this section, we investigate the blow-up property of the solutions to Problem (1.1) and prove Theorem 1.2. The main method is constructing a blowing-up lower solution and using the comparison principle to achieve our purpose.

Proof of TheOREM 1.3. (i) When $\alpha \beta>m n(p-1)(q-1)$ and the initial data is large enough, set

$$
\begin{aligned}
& \underline{u}(x, t)=(\tau-t)^{-\gamma_{1}} V_{1}(\xi), \quad \xi=|x|(\tau-t)^{-\ell_{1}}, \quad V_{1}(\xi)=\left(1+\frac{A}{2}-\frac{\xi^{2}}{2 A}\right)_{+}^{1 / m}, \\
& \underline{v}(x, t)=(\tau-t)^{-\gamma_{2}} V_{2}(\eta), \quad \eta=|x|(\tau-t)^{-\ell_{2}}, \quad V_{2}(\eta)=\left(1+\frac{A}{2}-\frac{\eta^{2}}{2 A}\right)_{+}^{1 / n},
\end{aligned}
$$

where $\gamma_{i}, \ell_{i}>0(i=1,2), A>1$ and $0<\tau<1$ are parameters to be determined. It is easy to see that $\underline{u}(x, t), \underline{v}(x, t)$ blow up at time $\tau$, so it enough to prove that $(\underline{u}(x, t), \underline{v}(x, t))$ is a lower solution of Problem (1.1). If we choose $\tau$ small enough such that

$$
\begin{aligned}
& \operatorname{supp} \underline{u}(\cdot, t)=\overline{B\left(0, R(\tau-t)^{\ell_{1}}\right)} \subset \overline{B\left(0, R \tau^{\ell_{1}}\right)} \subset \Omega, \\
& \operatorname{supp} \underline{v}(\cdot, t)=\overline{B\left(0, R(\tau-t)^{\ell_{2}}\right)} \subset \overline{B\left(0, R \tau^{\ell_{2}}\right)} \subset \Omega,
\end{aligned}
$$

where $R=(A(2+A))^{1 / 2}$, then $\left.\underline{u}(x, t)\right|_{\partial \Omega}=0,\left.\underline{v}(x, t)\right|_{\partial \Omega}=0$. Next if we choose the initial data large enough such that

$$
u_{0}(x) \geq \frac{1}{\tau^{\gamma_{1}}} V_{1}\left(\frac{|x|}{\tau^{\ell_{1}}}\right), \quad v_{0}(x) \geq \frac{1}{\tau^{\gamma_{2}}} V_{2}\left(\frac{|x|}{\tau^{\ell_{2}}}\right),
$$

then $(\underline{u}(x, t), \underline{v}(x, t))$ is a lower solution of Problem (1.1) if

$$
\begin{array}{r}
\underline{u}_{t}-\Delta_{m, p} \underline{u} \leq a \int_{\Omega} \underline{v}^{\alpha}(x, t) d x, \quad \underline{v}_{t}-\Delta_{n, q} \underline{v} \leq b \int_{\Omega} \underline{u}^{\beta}(x, t) d x, \\
(x, t) \in \Omega \times(0, \tau] .
\end{array}
$$

After a direct computation, we obtain

$$
\begin{aligned}
\underline{u}_{t} & =\frac{\gamma_{1} V_{1}(\xi)+\ell_{1} \xi V_{1}^{\prime}(\xi)}{(\tau-t)^{\gamma_{1}+1}}, & \underline{v}_{t} & =\frac{\gamma_{2} V_{2}(\eta)+\ell_{2} \eta V_{2}^{\prime}(\eta)}{(\tau-t)^{\gamma_{2}+1}}, \\
\nabla \underline{u}^{m} & =\frac{x}{A(\tau-t)^{m \gamma_{1}+2 \ell_{1}}}, & -\Delta \underline{u}^{m} & =\frac{N}{A(\tau-t)^{m \gamma_{1}+2 \ell_{1}}}, \\
\nabla \underline{v}^{n} & =\frac{x}{A(\tau-t)^{n \gamma_{2}+2 \ell_{2}}}, & -\Delta \underline{v}^{n} & =\frac{N}{A(\tau-t)^{n \gamma_{2}+2 \ell_{2}}},
\end{aligned}
$$

and

$$
\begin{aligned}
\Delta_{m, p} \underline{u} & =\left|\nabla \underline{u}^{m}\right|^{p-2} \Delta \underline{u}^{m}+(p-2)\left|\nabla \underline{u}^{m}\right|^{p-4}\left(\nabla \underline{u}^{m}\right)^{\tau} \cdot\left(H_{x}\left(\underline{u}^{m}\right)\right) \cdot \nabla \underline{u}^{m} \\
& =\left|\nabla \underline{u}^{m}\right|^{p-2} \Delta \underline{u}^{m}+(p-2)\left|\nabla \underline{u}^{m}\right|^{p-4} \sum_{j=1}^{N} \sum_{i=1}^{N} \frac{\partial \underline{u}^{m}}{\partial x_{i}} \frac{\partial^{2} \underline{u}^{m}}{\partial x_{i} x_{j}} \frac{\partial \underline{u}^{m}}{\partial x_{j}}
\end{aligned}
$$




$$
\begin{aligned}
\Delta_{n, q} \underline{v} & =\left|\nabla \underline{v}^{n}\right|^{q-2} \Delta \underline{v}^{n}+(q-2)\left|\nabla \underline{v}^{n}\right|^{q-4}\left(\nabla \underline{v}^{n}\right)^{\tau} \cdot\left(H_{x}\left(\underline{v}^{n}\right)\right) \cdot \nabla \underline{v}^{n} \\
& =\left|\nabla \underline{v}^{n}\right|^{q-2} \Delta \underline{v}^{n}+(q-2)\left|\nabla \underline{v}^{n}\right|^{q-4} \sum_{j=1}^{N} \sum_{i=1}^{N} \frac{\partial \underline{v}^{n}}{\partial x_{i}} \frac{\partial^{2} \underline{v}^{n}}{\partial x_{i} x_{j}} \frac{\partial \underline{v}^{n}}{\partial x_{j}},
\end{aligned}
$$

where $H_{x}\left(\underline{u}^{m}\right), H_{x}\left(\underline{v}^{n}\right)$ denote the Hessian matrix of $\underline{u}^{m}(x, t), \underline{v}^{n}(x, t)$, respectively.

Use the notation $d(\Omega)=\operatorname{diam}(\Omega)$, then from (4.2) and (4.3), we obtain

$$
\begin{aligned}
\left|\Delta_{m, p} \underline{u}\right| \leq & \frac{N}{A(\tau-t)^{m \gamma_{1}+2 \ell_{1}}}\left(\frac{d(\Omega)}{(\tau-t)^{m \gamma_{1}+2 \ell_{1}}}\right)^{p-2} \\
& +(p-2)\left(\frac{d(\Omega)}{(\tau-t)^{m \gamma_{1}+2 \ell_{1}}}\right)^{p-4}\left(\frac{d(\Omega)}{(\tau-t)^{m \gamma_{1}+2 \ell_{1}}}\right)^{2} \frac{N}{A(\tau-t)^{m \gamma_{1}+2 \ell_{1}}} \\
= & \frac{N(p-1)(d(\Omega))^{p-2}}{A(\tau-t)^{\left(m \gamma_{1}+2 \ell_{1}\right)(p-1)}}
\end{aligned}
$$

Similarly, from (4.2) and (4.4) we obtain

$$
\begin{aligned}
\left|\Delta_{n, q} \underline{v}\right| \leq & \frac{N}{A(\tau-t)^{n \gamma_{2}+2 \ell_{2}}}\left(\frac{d(\Omega)}{(\tau-t)^{n \gamma_{2}+2 \ell_{2}}}\right)^{q-2} \\
& +(q-2)\left(\frac{d(\Omega)}{(\tau-t)^{n \gamma_{2}+2 \ell_{2}}}\right)^{q-4}\left(\frac{d(\Omega)}{(\tau-t)^{n \gamma_{2}+2 \ell_{2}}}\right)^{2} \frac{N}{A(\tau-t)^{n \gamma_{2}+2 \ell_{2}}} \\
= & \frac{N(q-1)(d(\Omega))^{q-2}}{A(\tau-t)^{\left(n \gamma_{2}+2 \ell_{2}\right)(q-1)}} .
\end{aligned}
$$

Next, we compute the nonlocal term of (4.1)

$$
\begin{aligned}
& a \int_{\Omega} \underline{v}^{\alpha}(x, t) d x=\frac{a}{(\tau-t)^{\alpha \gamma_{2}}} \int_{B\left(0, R(\tau-t)^{\left.\ell_{2}\right)}\right.} V_{2}^{\alpha}\left(\frac{|x|}{(\tau-t)^{\ell_{2}}}\right) d x=\frac{a M_{1}}{(\tau-t)^{\alpha \gamma_{2}-N \ell_{2}}}, \\
& b \int_{\Omega} \underline{u}^{\beta}(x, t) d x=\frac{b}{(\tau-t)^{\beta \gamma_{1}}} \int_{B\left(0, R(\tau-t)^{\left.\ell_{1}\right)}\right.} V_{1}^{\beta}\left(\frac{|x|}{(\tau-t)^{\ell_{1}}}\right) d x=\frac{b M_{2}}{(\tau-t)^{\beta \gamma_{1}-N \ell_{1}}}
\end{aligned}
$$

where

$$
M_{1}=\int_{B(0, R)} V_{2}^{\alpha}(|\eta|) d \eta, \quad M_{2}=\int_{B(0, R)} V_{1}^{\beta}(|\xi|) d \xi
$$

If $0 \leq \xi, \eta \leq A$, then $1 \leq V_{1}(\xi) \leq(1+A / 2)^{1 / m}, 1 \leq V_{2}(\eta) \leq(1+A / 2)^{1 / n}$ and $V_{1}^{\prime}(\xi) \leq 0, V_{2}^{\prime}(\eta) \leq 0$. Combining the above inequalities and the definition of $M_{1}$ and $M_{2}$, we obtain

$$
\begin{aligned}
& M_{1}=\int_{B(0, R)} V_{2}^{\alpha}(|\eta|) d \eta \geq \int_{B(0, A)} V_{2}^{\alpha}(|\eta|) d \eta \geq\|B(0, A)\|, \\
& M_{2}=\int_{B(0, R)} V_{1}^{\beta}(|\xi|) d \xi \geq \int_{B(0, A)} V_{1}^{\beta}(|\xi|) d \xi \geq\|B(0, A)\| .
\end{aligned}
$$


Then from (4.2)-(4.8) we obtain

$$
\begin{aligned}
\underline{u}_{t}- & \Delta_{m, p} \underline{u}-a \int_{\Omega} \underline{v}^{\alpha}(x, t) d x \\
& \leq \frac{\gamma_{1}\left(1+\frac{A}{2}\right)^{1 / m}}{(\tau-t)^{\gamma_{1}+1}}+\frac{N(p-1)(d(\Omega))^{p-2}}{A(\tau-t)^{\left(m \gamma_{1}+2 \ell_{1}\right)(p-1)}}-\frac{a\|B(0, A)\|}{(\tau-t)^{\alpha \gamma_{2}-N \ell_{2}}}, \\
\underline{v}_{t}- & \Delta_{n, q} \underline{v}-b \int_{\Omega} \underline{u}^{\beta}(x, t) d x \\
& \leq \frac{\gamma_{2}\left(1+\frac{A}{2}\right)^{1 / n}}{(\tau-t)^{\gamma_{2}+1}}+\frac{N(q-1)(d(\Omega))^{q-2}}{A(\tau-t)^{\left(n \gamma_{2}+2 \ell_{2}\right)(q-1)}}-\frac{b\|B(0, A)\|}{(\tau-t)^{\beta \gamma_{1}-N \ell_{1}}} .
\end{aligned}
$$

If $\xi, \eta \geq A$, since $m, n \geq 1$, we obtain $V_{1}(\xi) \leq 1, V_{2}(\eta) \leq 1$ and $V_{1}^{\prime}(\xi) \leq-1 / m$, $V_{2}^{\prime}(\eta) \leq-1 / n$. Combining the above inequalities (4.2)-(4.7), and $M_{1} \geq 0, M_{2} \geq 0$, we obtain

$$
\begin{aligned}
\underline{u}_{t}-\Delta_{m, p} \underline{u}-a \int_{\Omega} \underline{v}^{\alpha}(x, t) d x & \leq \frac{\gamma_{1}-\frac{1}{m} \ell_{1} A}{(\tau-t)^{\gamma_{1}+1}}+\frac{N(p-1)(d(\Omega))^{p-2}}{A(\tau-t)^{\left(m \gamma_{1}+2 \ell_{1}\right)(p-1)}}, \\
\underline{v}_{t}-\Delta_{n, q} \underline{v}-b \int_{\Omega} \underline{u}^{\beta}(x, t) d x & \leq \frac{\gamma_{2}-\frac{1}{n} \ell_{2} A}{(\tau-t)^{\gamma_{2}+1}}+\frac{N(q-1)(d(\Omega))^{q-2}}{A(\tau-t)^{\left(n \gamma_{2}+2 \ell_{2}\right)(q-1)}} .
\end{aligned}
$$

If $0 \leq \xi \leq A$ and $\eta \geq A$, we have that (4.9) and (4.12) hold. If $\xi \geq A$ and $0 \leq \eta \leq A$, we have that (4.11) and (4.10) hold.

So, from the above discussions, (4.1) holds if the right-hand sides of (4.9)-(4.12) are nonpositive.

Since $p, q>2, m, n \geq 1$ and $\alpha \beta>m n(p-1)(q-1)>m n \geq 1$, we can choose two constants $\ell_{1}, \ell_{2}>0$ small enough such that

$$
\begin{aligned}
& \frac{1+\alpha+N\left(\ell_{2}+\alpha \ell_{1}\right)}{\alpha \beta-1}<\frac{1-2 \ell_{1}(p-1)}{m(p-1)-1}, \\
& \frac{1+\beta+N\left(\ell_{1}+\beta \ell_{2}\right)}{\alpha \beta-1}<\frac{1-2 \ell_{2}(q-1)}{n(q-1)-1} .
\end{aligned}
$$

Then we can choose two constants $\gamma_{1}, \gamma_{2}$ such that

$$
\begin{aligned}
& \frac{1+\alpha+N\left(\ell_{2}+\alpha \ell_{1}\right)}{\alpha \beta-1}<\gamma_{1}<\frac{1-2 \ell_{1}(p-1)}{m(p-1)-1}, \\
& \frac{1+\beta+N\left(\ell_{1}+\beta \ell_{2}\right)}{\alpha \beta-1}<\gamma_{2}<\frac{1-2 \ell_{2}(q-1)}{n(q-1)-1},
\end{aligned}
$$

that is

$$
\begin{gathered}
\left(m \gamma_{1}+2 \ell_{1}\right)(p-1)<\gamma_{1}+1<\alpha \gamma_{2}-N \ell_{2}, \\
\left(n \gamma_{2}+2 \ell_{2}\right)(q-1)<\gamma_{2}+1<\beta \gamma_{1}-N \ell_{1} .
\end{gathered}
$$


Furthermore, if we choose $A>\max \left\{1, m \gamma_{1} / \ell_{1}, n \gamma_{2} / \ell_{2}\right\}$, then for $\tau>0$ sufficiently small, the right-hand sides of (4.9)-(4.12) ar nonpositive, so (4.1) holds, and we obtain Theorem 1.3(i).

(ii) When $\alpha \beta=m n(p-1)(q-1)$ and $\Omega$ contains a sufficiently large ball, we assume that $0 \in \Omega$ and a ball $B(0, R) \subset \subset \Omega$. Then we only need to show that the radial solution of problem $(1.1)$ on $(\overline{B(0, R)} \times[0, T]) \times \overline{B(0, R)} \times[0, T])$ blows up in finite time.

Since $p, q>2$ and $\alpha \beta=m n(p-1)(q-1)$, we can choose two constants $\ell_{1}, \ell_{2}$ such that

$$
\frac{\alpha}{m(p-1)}=\frac{\ell_{1}}{\ell_{2}}=\frac{n(q-1)}{\beta}, \quad \text { that is, } \quad \ell_{2} \alpha=m(p-1) \ell_{1}, \ell_{1} \beta=n(q-1) \ell_{2} .
$$

Firstly, let us consider the following elliptic problem on $(0, R)$,

$$
\left\{\begin{array} { l } 
{ - \frac { d } { d r } ( r ^ { N - 1 } | \frac { d \varphi ^ { m } } { d r } | ^ { p - 2 } \frac { d \varphi ^ { m } } { d r } ) = r ^ { N - 1 } , } \\
{ \varphi ^ { \prime } ( 0 ) = 0 , \quad \varphi ( R ) = 0 , }
\end{array} \quad \left\{\begin{array}{l}
-\frac{d}{d r}\left(r^{N-1}\left|\frac{d \psi^{n}}{d r}\right|^{q-2} \frac{d \psi^{n}}{d r}\right)=r^{N-1}, \\
\psi^{\prime}(0)=0, \quad \psi(R)=0 .
\end{array}\right.\right.
$$

Then it is easy to show

$$
\begin{aligned}
& \varphi(r)=\left(\frac{p-1}{p}\right)^{1 / m}\left(\frac{1}{N}\right)^{1 / m(p-1)}\left(R^{p /(p-1)}-r^{p /(p-1)}\right)^{1 / m}, \\
& \psi(r)=\left(\frac{q-1}{q}\right)^{1 / n}\left(\frac{1}{N}\right)^{1 / n(q-1)}\left(R^{q /(q-1)}-r^{q /(q-1)}\right)^{1 / n} .
\end{aligned}
$$

By Assumption 1.1 on initial data, we can choose $s_{0}>0$ small enough that

$$
u_{0}(r) \geq s_{0}^{\ell_{1}} \varphi(r), \quad v_{0}(r) \geq s_{0}^{\ell_{2}} \psi(r), \quad \forall r \in[0, R) .
$$

Next, let us consider the following Cauchy problem with $s(0)=s_{0}$,

$$
\begin{aligned}
s^{\prime}(t) & =\min \left\{\frac{a c_{1}-1}{\ell_{1} M_{1}}, \frac{b c_{2}-1}{\ell_{2} M_{2}}\right\} s^{\Upsilon}(t), \\
\Upsilon & =\min \left\{m(p-1) \ell_{1}-\ell_{1}+1, n(q-1) \ell_{2}-\ell_{2}+1\right\},
\end{aligned}
$$

where for $R$ large enough and $\omega(N)$ is the volume of the unit ball in $N$-dimensional space

$$
\begin{aligned}
c_{1} & =\int_{B(0, R)} \psi^{\alpha}(|x|) d x \\
& =\int_{0}^{R} d r \int_{\partial B(0, r)} \psi^{\alpha}(r) d \sigma \\
& =\int_{0}^{R} N \omega(N) \psi^{\alpha}(r) r^{N-1} d r \\
& =N \omega(N)\left(\frac{q-1}{q}\right)^{\frac{\alpha}{n}}\left(\frac{1}{N}\right)^{\frac{\alpha}{n(q-1)}} \int_{0}^{R}\left(R^{\frac{q}{q-1}}-r^{\frac{q}{q-1}}\right)^{\frac{\alpha}{n}} r^{N-1} d r>\frac{1}{a},
\end{aligned}
$$




$$
\begin{aligned}
c_{2} & =\int_{B(0, R)} \varphi^{\beta}(|x|) d x \\
& =\int_{0}^{R} d r \int_{\partial B(0, r)} \varphi^{\beta}(r) d \sigma \\
& =\int_{0}^{R} N \omega(N) \varphi^{\beta}(r) r^{N-1} d r \\
& =N \omega(N)\left(\frac{p-1}{p}\right)^{\frac{\beta}{m}}\left(\frac{1}{N}\right)^{\frac{\beta}{m(p-1)}} \int_{0}^{R}\left(R^{\frac{p}{p-1}}-r^{\frac{p}{p-1}}\right)^{\frac{\beta}{m}} r^{N-1} d r>\frac{1}{b} \\
M_{1} & =\left(\frac{p-1}{p}\right)^{\frac{1}{m}}\left(\frac{1}{N}\right)^{\frac{1}{m(p-1)}} R^{\frac{p}{m(p-1)}}, \quad M_{2}=\left(\frac{q-1}{q}\right)^{\frac{1}{n}}\left(\frac{1}{N}\right)^{\frac{1}{n(q-1)}} R^{\frac{q}{n(q-1)}}
\end{aligned}
$$

Since $\Upsilon>1$, then there exists a constant $\tau^{*}$ such that $\lim _{t \rightarrow \tau^{*} S}(t)=+\infty$.

Finally, we construct $\underline{u}(r, t)=s^{\ell_{1}} \varphi(r)$ and $\underline{v}(r, t)=s^{\ell_{2}} \psi(r)$, then $(\underline{u}(r, t), \underline{v}(r, t))$ blows up in finite time. So it is enough to prove that $(\underline{u}(r, t), \underline{v}(r, t))$ is a lower solution of Problem $(1.1)$ on $(\overline{B(0, R)} \times[0, \tau]) \times(\overline{B(0, R)} \times[0, \tau])$. Let us make some simple computations:

$$
\begin{aligned}
\Delta_{m, p} \underline{u} & =\nabla \cdot\left(\left|\left(\underline{u}^{m}\right)_{r}\right|^{p-2}\left(\underline{u}^{m}\right)_{r} \frac{x}{r}\right) \\
& =\sum_{i=1}^{N}\left(\left|\left(\underline{u}^{m}\right)_{r}\right|^{p-2}\left(\underline{u}^{m}\right)_{r} \frac{x_{i}}{r}\right)_{x_{i}} \\
& =\sum_{i=1}^{N}\left(\left|\left(\underline{u}^{m}\right)_{r}\right|^{p-2}\left(\underline{u}^{m}\right)_{r}\right)_{r} \frac{x_{i}^{2}}{r^{2}}+\sum_{i=1}^{N}\left|\left(\underline{u}^{m}\right)_{r}\right|^{p-2}\left(\underline{u}^{m}\right)_{r} \frac{r^{2}-x_{i}^{2}}{r^{3}} \\
& =\left(\left|\left(\underline{u}^{m}\right)_{r}\right|^{p-2}\left(\underline{u}^{m}\right)_{r}\right)_{r}+\left|\left(\underline{u}^{m}\right)_{r}\right|^{p-2}\left(\underline{u}^{m}\right)_{r} \frac{N-1}{r} \\
& =r^{1-N}\left(r^{N-1}\left|\left(\underline{u}^{m}\right)_{r}\right|^{p-2}\left(\underline{u}^{m}\right)_{r}\right)_{r}:=\mathfrak{s}(\underline{u}), \\
\Delta_{n, q} \underline{v} & =r^{1-N}\left(r^{N-1}\left|\left(\underline{v}^{n}\right)_{r}\right|^{q-2}\left(\underline{v}^{n}\right)_{r}\right)_{r}:=\Im(\underline{v}) .
\end{aligned}
$$

Then Problem (1.1) becomes the following equations,

$$
\begin{aligned}
\underline{u}_{t}- & \Im(\underline{u})-a \int_{B(0, R)} \underline{v}^{\alpha}(|x|) d x \\
& =\ell_{1} \varphi s^{\ell_{1}-1} s^{\prime}(t)+s^{m(p-1) \ell_{1}}(t)-a c_{1} s^{\alpha \ell_{2}}(t) \\
& =\varphi s^{\ell_{1}-1}\left(\ell_{1} s^{\prime}(t)+\varphi^{-1} s^{m(p-1) \ell_{1}-\ell_{1}+1}(t)-a c_{1} \varphi^{-1} s^{\alpha \ell_{2}-\ell_{1}+1}(t)\right) \\
& =\varphi s^{\ell_{1}-1}\left(\ell_{1} s^{\prime}(t)-\left(a c_{1}-1\right) \varphi^{-1} s^{m(p-1) \ell_{1}-\ell_{1}+1}(t)\right) \\
& \leq \varphi s^{\ell_{1}-1}\left(\ell_{1} s^{\prime}(t)-\left(a c_{1}-1\right) M_{1}^{-1} s^{m(p-1) \ell_{1}-\ell_{1}+1}(t)\right) \\
& \leq 0, \quad \forall(r, t) \in B(0, R) \times(0, \tau),
\end{aligned}
$$




$$
\begin{aligned}
& \underline{v}_{t}-\Im(\underline{v})-b \int_{B(0, R)} \underline{u}^{\beta}(|x|) d x \\
& =\ell_{2} \psi s^{\ell_{2}-1} s^{\prime}(t)+s^{n(q-1) \ell_{2}}(t)-b c_{2} s^{\beta \ell_{1}}(t) \\
& =\psi s^{\ell_{2}-1}\left(\ell_{2} s^{\prime}(t)+\psi^{-1} s^{n(q-1) \ell_{2}-\ell_{2}+1}(t)-b c_{2} \psi^{-1} s^{\beta \ell_{1}-\ell_{2}+1}(t)\right) \\
& =\psi s^{\ell_{2}-1}\left(\ell_{2} s^{\prime}(t)-\left(b c_{2}-1\right) \psi^{-1} s^{n(q-1) \ell_{2}-\ell_{2}+1}(t)\right) \\
& \leq \psi s^{\ell_{2}-1}\left(\ell_{2} s^{\prime}(t)-\left(b c_{2}-1\right) M_{2}^{-1} s^{n(q-1) \ell_{2}-\ell_{2}+1}(t)\right) \\
& \leq 0, \quad \forall(r, t) \in B(0, R) \times(0, \tau), \\
& \left.r^{N-1}\left|\left(\underline{u}^{m}\right)_{r}\right|^{p-2}\left(\underline{u}^{m}\right)_{r}\right|_{r=0}=0,\left.\quad r^{N-1}\left|\left(\underline{v}^{n}\right)_{r}\right|^{q-2}\left(\underline{v}^{n}\right)_{r}\right|_{r=0}=0, \quad \forall t \in[0, \tau], \\
& \underline{u}(R, t)=s^{\ell_{1}} \varphi(R)=0, \quad \underline{v}(R, t)=s^{\ell_{2}} \psi(R)=0, \quad \forall t \in[0, \tau], \\
& \underline{u}(r, 0)=s_{0}^{\ell_{1}} \varphi(r) \leq u_{0}(r), \quad \underline{u}(r, 0)=s_{0}^{\ell_{2}} \psi(r) \leq v_{0}(r), \quad \forall r \in[0, R] .
\end{aligned}
$$

So, $(\underline{u}(r, t), \underline{v}(r, t))$ is a lower solution of Problem $(1.1)$ on $(\overline{B(0, R)} \times[0, \tau])$ $\times(\overline{B(0, R)} \times[0, \tau])$, we obtain Theorem 1.3(ii). The proof of Theorem 1.3 is complete.

\section{The special case $\alpha=n(q-1), \beta=m(p-1)$}

In this section we consider Problem (1.1) for a special case $\alpha=n(q-1)$, $\beta=m(p-1)$, similar to Sections 3 and 4 , we prove Theorem 1.4 by constructing special upper and lower solutions.

5.1. Global existence In this section we prove the conclusion (i) of Theorem 1.4. Since $\lambda \mu \leq(a b)^{-1}$, we can take two positive constants $\Lambda_{1}, \Lambda_{2}$ large enough such that

$$
a \mu \leq \frac{\Lambda_{1}^{m(p-1)}}{\Lambda_{2}^{n(q-1)}} \leq(b \lambda)^{-1}, \quad \Lambda_{1} \zeta(x) \geq u_{0}(x), \quad \Lambda_{2} \vartheta(x) \geq v_{0}(x) .
$$

Set $\bar{u}(x, t)=\Lambda_{1} \zeta(x), \bar{v}(x, t)=\Lambda_{2} \vartheta(x)$, then we show that $(\bar{u}(x, t), \bar{v}(x, t))$ is a upper solution of Problem (1.1), which exists globally. After a simple computation, we obtain

$$
\begin{aligned}
& \bar{u}_{t}-\Delta_{m, p} \bar{u}-a \int_{\Omega} \bar{v}^{n(q-1)} d x=\Lambda_{1}^{m(p-1)}-a \mu \Lambda_{2}^{n(q-1)} \geq 0 \\
& \bar{v}_{t}-\Delta_{n, q} \bar{v}-b \int_{\Omega} \bar{u}^{m(p-1)} d x=\Lambda_{2}^{n(q-1)}-b \lambda \Lambda_{1}^{m(p-1)} \geq 0 .
\end{aligned}
$$

Noting that $\bar{u}(x, t)=\bar{v}(x, t)=0$ on $\partial \Omega \times[0,+\infty)$, we obtain that $(\bar{u}(x, t), \bar{v}(x, t))$ is an upper solution of Problem (1.1). Then conclusion (i) of Theorem 1.4 holds.

5.2. Blow-up In this section we prove conclusion (ii) of Theorem 1.4. First, we introduce the following useful lemma. 
LEMMA 5.1. Suppose that the initial data $\left(u_{0}(x), v_{0}(x)\right)$ satisfies the Assumption 1.1 and $\lambda \mu>(a b)^{-1}$, then there exist two positive constants $\sigma_{1}, \sigma_{2}$ such that

$$
u(x, t) \geq \sigma_{1} \zeta(x), \quad v(x, t) \geq \sigma_{2} \vartheta(x), \quad \forall(x, t) \in \bar{Q}_{T}
$$

PROOF. Since $\lambda \mu>(a b)^{-1}$, we can take two appropriate $\sigma_{1}, \sigma_{2}$ positive constants such that

$$
a \mu \geq \frac{\sigma_{1}^{m(p-1)}}{\sigma_{2}^{n(q-1)}} \geq(b \lambda)^{-1}, \quad \sigma_{1} \zeta(x) \leq u_{0}(x), \quad \sigma_{2} \vartheta(x) \leq v_{0}(x)
$$

Let $\underline{u}(x, t)=\sigma_{1} \zeta(x), \underline{v}(x, t)=\sigma_{2} \vartheta(x)$, then we will show $(\underline{u}(x, t), \underline{v}(x, t))$ is a lower solution of Problem (1.1). After a simple computation, we obtain

$$
\begin{aligned}
& \underline{u}_{t}-\Delta_{m, p} \underline{u}-a \int_{\Omega} \underline{v}^{n(q-1)} d x=\sigma_{1}^{m(p-1)}-a \mu \sigma_{2}^{n(q-1)} \leq 0, \\
& \underline{v}_{t}-\Delta_{n, q} \underline{v}-b \int_{\Omega} \underline{u}^{m(p-1)} d x=\sigma_{2}^{n(q-1)}-b \lambda \sigma_{1}^{m(p-1)} \leq 0 .
\end{aligned}
$$

Noting that $\underline{u}(x, t)=\underline{v}(x, t)=0$, we obtain that $(\underline{u}(x, t), \underline{v}(x, t))$ is a lower solution of Problem (1.1). The proof of Lemma 5.1 is complete.

Now we can prove Theorem 1.4(ii). For $\Omega_{1} \subset \subset \Omega$, let us consider the following elliptic equation,

$$
\left\{\begin{array} { l l } 
{ - \Delta _ { m , p } \zeta _ { 1 } = 1 , } & { x \in \Omega _ { 1 } , } \\
{ \zeta _ { 1 } = 0 , } & { x \in \partial \Omega _ { 1 } , }
\end{array} \quad \left\{\begin{array}{ll}
-\Delta_{n, q} \vartheta_{1}=1, & x \in \Omega_{1}, \\
\vartheta_{1}=0, & x \in \partial \Omega_{1} .
\end{array}\right.\right.
$$

Then the comparison principle asserts that $\left.\zeta(x)\right|_{\Omega_{1}} \geq \zeta_{1}(x),\left.\vartheta(x)\right|_{\Omega_{1}} \geq \vartheta_{1}(x)$. Take

$$
\lambda_{1}=\int_{\Omega} \zeta_{1}^{m(p-1)}(x) d x, \quad \mu_{1}=\int_{\Omega} \vartheta_{1}^{n(q-1)}(x) d x .
$$

Since $\lambda \mu>(a b)^{-1}$ and $\left.\zeta(x)\right|_{\partial \Omega}=0,\left.\vartheta(x)\right|_{\partial \Omega}=0$, we can choose some $\Omega_{1}$ such that $\lambda_{1} \mu_{1}>(a b)^{-1}$. From Lemma 5.1, we can see $\left.u(x, t)\right|_{\Omega_{1}} \geq \sigma_{1} \zeta_{1}(x),\left.v(x, t)\right|_{\Omega_{1}} \geq$ $\sigma_{2} \vartheta_{1}(x)$.

Next let us take a domain $\Omega_{2} \subset \subset \Omega_{1}$ and use the notation

$$
\varepsilon=\min \left\{\inf _{x \in \bar{\Omega}_{2}} \sigma_{1} \zeta_{1}(x), \inf _{x \in \bar{\Omega}_{2}} \sigma_{2} \vartheta_{1}(x)\right\}>0 .
$$

Then,

$$
\left.u(x, t)\right|_{\bar{\Omega}_{2}} \geq\left.\sigma_{1} \zeta_{1}(x)\right|_{\bar{\Omega}_{2}} \geq \varepsilon,\left.\quad v(x, t)\right|_{\bar{\Omega}_{2}} \geq\left.\sigma_{2} \vartheta_{1}(x)\right|_{\bar{\Omega}_{2}} \geq \varepsilon
$$


So, the above discussion ensures that the solution $(u(x, t), v(x, t))$ of Problem (1.1) is an upper solution of the following problem in $\left(\bar{\Omega}_{2} \times[0, T]\right) \times\left(\bar{\Omega}_{2} \times[0, T]\right)$,

$$
\begin{cases}\underline{u}_{t}-\Delta_{m, p} \underline{u}=a \int_{\Omega} \underline{v}^{\alpha}(x, t) d x, & (x, t) \in \Omega_{2} \times(0, T], \\ \underline{v}_{t}-\Delta_{n, q} \underline{v}=b \int_{\Omega} \underline{u}^{\beta}(x, t) d x, & (x, t) \in \Omega_{2} \times(0, T], \\ \underline{u}(x, t)=\varepsilon, \quad \underline{v}(x, t)=\varepsilon, & (x, t) \in \partial \Omega_{2} \times(0, T], \\ \underline{u}(x, 0)=\varepsilon, \quad \underline{v}(x, 0)=\varepsilon, & x \in \Omega_{2} .\end{cases}
$$

Denote $\wp=\max \left\{\sup _{x \in \bar{\Omega}_{2}} \zeta_{1}(x), \sup _{x \in \bar{\Omega}_{2}} \vartheta_{1}(x)\right\}$, and consider the following Cauchy problem,

$$
\begin{array}{ccc}
\wp s_{1}^{\prime}(t)+s_{1}^{m(p-1)}-a \mu_{1} s_{2}^{n(q-1)}(t)=0, & s_{1}(0)=\varepsilon / \wp \\
\wp s_{2}^{\prime}(t)+s_{2}^{n(q-1)}-b \lambda_{1} s_{1}^{m(p-1)}(t)=0, & s_{2}(0)=\varepsilon / \wp .
\end{array}
$$

Multiplying the first equation of (5.2) by $b \lambda_{1}+1$, the second equation of (5.2) by $a \mu_{1}+1$ and combining them together, we obtain

$$
\wp\left(b \lambda_{1}+1\right) s_{1}^{\prime}(t)+\wp\left(a \mu_{1}+1\right) s_{2}^{\prime}(t)=\left(a b \lambda_{1} \mu_{1}-1\right)\left(s_{1}^{m(p-1)}+s_{2}^{n(q-1)}\right) .
$$

Since $m(p-1)>m \geq 1, n(q-1)>n \geq 1$ and $a b \lambda_{1} \mu_{1}>1$, there exists a constant $T^{\prime}<+\infty$ such that

$$
\lim _{t \rightarrow T^{\prime}}\left(s_{1}(t)+s_{2}(t)\right)=+\infty .
$$

Set $\tilde{u}(x, t)=s_{1}(t) \zeta_{1}(x), \widetilde{v}(x, t)=s_{2}(t) \vartheta_{1}(x)$, then $(\tilde{u}(x, t), \widetilde{v}(x, t))$ blows up in finite time. So, the solution of Problem (5.1) blows up in finite time if $(\tilde{u}(x, t), \widetilde{v}(x, t))$ is a lower solution of Problem (5.1). After a simple computation, we obtain

$$
\begin{aligned}
& \tilde{u}_{t}-\Delta_{m, p} \tilde{u}-a \int_{\Omega} \widetilde{v}^{\alpha}(x, t) d x \\
& =\zeta_{1}(x) s_{1}^{\prime}(t)+s_{1}^{m(p-1)}-a \mu_{1} s_{2}^{n(q-1)}(t) \\
& \leq \wp s_{1}^{\prime}(t)+s_{1}^{m(p-1)}-a \mu_{1} s_{2}^{n(q-1)}(t)=0, \quad \forall(x, t) \in \Omega_{2} \times(0, T], \\
& \tilde{v}_{t}-\Delta_{n, q} \tilde{u}-b \int_{\Omega} \widetilde{u}^{\beta}(x, t) d x \\
& =\vartheta_{1}(x) s_{2}^{\prime}(t)+s_{2}^{n(q-1)}-b \lambda_{1} s_{1}^{m(p-1)}(t) \\
& \leq \wp s_{2}^{\prime}(t)+s_{2}^{n(q-1)}-b \lambda_{1} s_{2}^{m(p-1)}(t)=0, \quad \forall(x, t) \in \Omega_{2} \times(0, T], \\
& \tilde{u}(x, t)=s_{1}(t) \zeta_{1}(x)=0, \quad \widetilde{v}(x, t)=s_{2}(t) \vartheta_{1}(x)=0, \forall(x, t) \in \partial \Omega_{2} \times[0, T], \\
& \tilde{u}(x, 0)=s_{1}(0) \zeta_{1}(x) \leq \varepsilon, \quad \widetilde{v}(x, 0)=s_{2}(0) \vartheta_{1}(x) \leq \varepsilon, \forall x \in \Omega_{2} \text {. }
\end{aligned}
$$

So, $\widetilde{u}(x, t), \widetilde{v}(x, t))$ is a lower solution of Problem (5.1). Then the conclusion of Theorem 1.4(ii) holds. The proof of Theorem 1.4 is complete. 


\section{Acknowledgements}

This work is supported in part by NNSF of China (10771226), and in part by the Natural Science Foundation Project of CQ CSTC (2007BB0124).

\section{References}

[1] J. R. Anderson and K. Deng, "Global existence for degenerate parabolic equations with a non-local forcing", Math. Anal. Methods Appl. Sci. 20 (1997) 1069-1087.

[2] M. F. Bidanut-Véon and M. Garciá-Huidobro, "Regular and singular solutions of a quasilinear equation with weights", Asymptot. Anal. 28 (2001) 115-150.

[3] W. B. Deng, "Global existence and finite time blow up for a degenerate reaction-diffusion system", Nonlinear Anal. 60 (2005) 977-991.

[4] K. Deng and H. A. Levine, "The role of critical exponents in blow-up theorems: the sequel", $J$. Math. Anal. Appl. 243 (2000) 85-126.

[5] W. B. Deng, Y. X. Li and C. H. Xie, "Blow-up and global existence for a nonlocal degenerate parabolic system”, J. Math. Anal. Appl. 277 (2003) 199-217.

[6] A. de Pablo, F. Quiros and J. D. Rossi, "Asymptotic simplification for a reaction-diffusion problem with a nonlinear boundary condition", IMA J. Appl. Math. 67 (2002) 69-98.

[7] J. I. Díaz, "Nonlinear partial differential equations and free boundaries", in Elliptic equations, Volume 1 (Pitman, London, 1985).

[8] E. Dibenedetto, Degenerate parabolic equations (Springer, Berlin, 1993).

[9] L. L. Du, "Blow-up for a degenerate reaction-diffusion system with nonlinear nonlocal sources", J. Comput. Appl. Math. 202 (2007) 237-247.

[10] Z. W. Duan, W. B. Deng and C. H. Xie, "Uniform blow-up profile for a degenerate parabolic system with nonlocal source", Comput. Math. Appl. 47 (2004) 977-995.

[11] V. A. Galaktionov, S. P. Kurdyumov and A. A. Samarskii, "A parabolic system of quasi-linear equations I", Differ. Equ. 19 (1983) 1558-1571.

[12] V. A. Galaktionov, S. P. Kurdyumov and A. A. Samarskii, "A parabolic system of quasi-linear equations II", Differ. Equ. 21 (1985) 1049-1062.

[13] V. A. Galaktionov and H. A. Levine, "On critical Fujita exponents for heat equations with nonlinear flux conditions on the boundary", Israel J. Math. 94 (1996) 125-146.

[14] V. A. Galaktionov and J. L. Vázquez, "The problem of blow-up in nonlinear parabolic equations", Dist. Cont. Dyn. Systems 8 (2002) 399-433.

[15] H. Ishii, "Asymptotic stability and blowing up of solutions of some nonlinear equations", $J$. Differential Equations 26 (1997) 291-319.

[16] A. S. Kalashnikov, "Some problems of the qualitative theory of nonlinear degenerate parabolic equations of second order", Russian Math. Surveys 42 (1987) 169-222.

[17] H. A. Levine, "The role of critical exponents in blow-up theorems", SIAM Rev. 32 (1990) 262-288.

[18] H. A. Levine and L. E. Payne, "Nonexistence theorems for the heat equation with nonlinear boundary conditions for the porous medium equation backward in time", J. Differential Equations 16 (1974) 319-334.

[19] F. C. Li and C. H. Xie, "Global and blow-up solutions to a $p$-Laplacian equation with nonlocal source", Comput. Math. Appl. 46 (2003) 1525-1533.

[20] F. C. Li and C. H. Xie, "Global existence and blow-up for a nonlinear porous medium equation", Appl. Math. Lett. 16 (2003) 185-192.

[21] F. Quiros and J. D. Rossi, "Blow-up sets and Fujita type curves for a degenerate parabolic system with nonlinear boundary conditions", Indiana Univ. Math. J. 50 (2001) 629-654.

[22] A. A. Samarskii, V. A. Galaktionov, S. P. Kurdyumov and A. P. Mikhailov, Blow-up in quasilinear parabolic equations (Walter de Gruyter, Berlin, 1985).

[23] W. J. Sun and S. Wang, "Nonlinear degenerate parabolic equation with nonlinear boundary condition”, Acta Math. Sin. (Engl. Ser.) 21 (2005) 847-854. 
[24] M. Tsutsumi, "Existence and nonexistence of global solutions for nonlinear parabolic equations", Publ. Res. Inst. Math. Sci. 8 (1972) 221-229.

[25] J. L. Vázquez, The porous medium equations: mathematical theory (Clarendon Press, Oxford, 2007).

[26] S. Wang, "Doubly nonlinear degenerate parabolic systems with coupled nonlinear boundary conditions", J. Differential Equations 182 (2002) 431-469.

[27] Z. Q. Wu, J. N. Zhao, J. X. Yin and H. L. Li, Nonlinear diffusion equations (Word Scientific, River Edge, NJ, 2001).

[28] J. Zhao, "Existence and nonexistence of solutions for $u_{t}-\nabla \cdot\left(|\nabla u|^{p-2} \nabla u\right)=f(\nabla u, u, x, t)$ ", J. Math. Anal. Appl. 173 (1993) 130-146.

[29] S. N. Zheng, X. F. Song and Z. X. Jiang, "Critical Fujita exponents for degenerate parabolic equations coupled via nonlinear boundary flux", J. Math. Anal. Appl. 298 (2004) 308-324.

[30] J. Zhou and C. L. Mu, "On critical Fujita exponent for degenerate parabolic system coupled via nonlinear boundary flux", Proc. Edinb. Math. Soc. 51 (2008) 785-805.

[31] J. Zhou and C. L. Mu, "The critical curve for a non-Newtonian polytropic filtration system coupled via nonlinear boundary flux", Nonlinear Anal. 68 (2008) 1-11. 